Conclusions Capacity building efforts, like high-level in-service courses and interactive workshops have enabled interested parties to make evidence-based recommendations and decisions on the introduction of any new vaccine, and to successfully implement new vaccine introduction.

\section{PA-112 INTRODUCTION OF A NEW VACCINE INTO NATIONAL IMMUNISATION PROGRAMMES IN AFRICA: THE ROLE OF CAPACITY BUILDING}

Carine Dochez, ${ }^{1}$ Rosemary Burnett, ${ }^{2}$ M. Jeffrey Mphahlele ${ }^{3}$. ${ }^{1} N E S I$, Antwerp, Belgium; ${ }^{2}$ SAVIC, South Africa; ${ }^{3} M R C$, South Africa

10.1136/bmjgh-2016-000260.141

Background Members of National Immunisation Technical Advisory Groups, policy-makers, EPI managers and vaccinators are tasked with making evidence-based recommendations and decisions on whether a new vaccine merits introduction into national immunisation programmes; implementation of new vaccine introduction; and efficient management of immunisation programmes. Therefore it is paramount that they are equipped with the latest state-of-the-art information on vaccines and immunisation.

Methods Capacity building activities - such as high-level in-service vaccinology courses, other interactive courses and workshops (mid-level management training and experience exchange workshops) - address all steps required for decisionmaking on new vaccine introduction into national immunisation programmes. These include establishing: 1) burden of disease to be prevented; 2) existence of a good intervention (i.e. is the vaccine efficacious, safe and acceptable for the target population); 3) the cost of the new vaccine, its implementation and the comparative effectiveness with other vaccines/interventions in terms of health gains; 4) whether finances to pay for the new vaccine are available; and 5) programmatic implications. Interested parties are trained on this rational decision-making process to be followed before embarking on new vaccine introduction, on key implementation steps, and efficient management of immunisation programmes.

Results Several inter-country vaccinology courses and interactive workshops, which were organised during the last years in the African region (e.g. Kenya and South Africa), will be presented in detail. These capacity building activities have contributed to successful introduction of new vaccines in the African region, key ones being rotavirus, pneumococcal and currently human papillomavirus vaccines. This concerted effort has contributed for example to the successful introduction of rotavirus vaccine in 29 African countries to date. 